\section{THE THYROID IN RELATION TO OTHER ENDOCRINE GLANDS}

\author{
By L. R. Broster, F.R.C.S: \\ (Surg., Charing Cross Hosp., etc.)
}

In this series of articles on the diseases of the thyroid gland I have been asked to write an article dealing with the relationship of the thyroid gland to the rest of the Endocrine System, showing how it may affect other ductless glands, and in its turn be affected by them. It would seem that an article of this kind should be written by a physician or an experimental endocrinologist rather than by a general surgeon, whose time and energies are devoted more to the operative treatment of the fully established stages of thyroid disease. Intercurrent disturbances of a polyglandular nature are often incidental in these cases, and perhaps the only advantage enjoyed by the surgeon is their study under the stress and strain of operative procedure. These primary and secondary interactions are extremely difficult to interpret or assess. Indeed, the same is true of the whole subject of Endocrinology where, owing to the limitations imposed on clinical science, medical research has had to resort more and more to animal experimentation.

This wide and growing field has added much to our knowledge, and has been the chief source not only of our observations on interglandular reactions, but has given us useful tests, such as the Aschheim Zondek test for pregnancy and the Capon test for male sex hormone, which are directly applicable to clinical advantage. On the other hand this mode of investigation has the inherent defect that it deals with species in different stages of evolution, and the results are apt to be confusing and conflicting. For instance, if tadpoles are fed on thyroid it hastens their metamorphosis, but produces dwarf frogs; whereas removal of the thyroid gland in young goats and puppies retards their growth, a condition which corresponds to cretinism in children.

Before proceeding, let us for a moment consider the position of the thyroid gland from the evolutionary point of view. There is much to be said in favour of the increasing rôle of importance played by the thyroid in the rise of man from the lower animals. Crile made an important observation in correlating the relative size and weight of the thyroid and adrenal glands in different species of animals. For instance, the size of the adrenal to the thyroid is greater in aggressive types of animal, such as the lion, than it is in the more defensive types, such as the antelope; whereas in the sluggish types, like the alligator, there is little or no difference. This ratio is entirely upset in man, where, except in the foetal stage, the thyroid is much bigger than the adrenals. Consequently in losing many of his animal attributes, such as muscular strength, energy, agility and endurance; by the dwindling in acuity of his special senses, as that of hearing and smelling; by contraction of his visual fields, although this is compensated for by binocular vision; by a diminution of the aural prima, and by a loss in the quality of his teeth, man might have become a sluggish animal, had it not been for those attributes that we associate with the thyroid gland, which have also been accompanied by the rise of the cerebral cortex.

These attributes of the thyroid are correlated with a restless activity and a heightened degree and range of metabolism. Another feature in the function of the thyroid gland is foreshadowed by its anatomical development from the buccal cavity, a feature which it also shares with the anterior lobe of the pituitary gland. Their common origin suggests that they were primarily designed for digestive purposes. It is therefore not surprising that these two glands together should have become primarily responsible for the promotion of normal growth and development.

\section{Functions of the Thyroid Gland.}

- In a short paper of this kind it is only possible to make a broad survey of the more importante functions of the thyroid gland. No gland of the endocrine series presents such a clear and contrasting clinical picture as the thyroid, both in its overactive state-as in Graves' Disease-or in its absent or deficient state-myxoedema in the adult or cretinism in the young. It produces only one hormone, thyroxin, a compound which belongs to the Tyrosine Group and possesses an iodine linkage.

Let us first consider its overactive state, first described by Graves in 1835 . True hyperthyroidism is characterised by three primary signs: enlargement of the thyroid, exophthalmos and tachycardia. To this list there are added more variable secondary symptoms, such as muscular incoordination with tremors, vasomotor instability with sweating and blushing, the skin is moist and slightly bronzed, there may be diarrhoea or vomiting. These symptoms are characterised by their waxing and waning and so are phasic in type. In the later stages there is wasting, muscular weakness, depression of the sex function with infertility and amenorrhoea. Mental changes are common. These patients are apt to be excitable, over-anxious and emotionally unstable, and give a general impression of fear and alertness. These mental states are improved by operation, but the more severe obsessional neuroses and depressions generally remain

Graves' Disease occurs predominantly in women, 
and a familial incidence is common. The writer's experience suggests that it is a war disease. Its incidence and severity have increased and fluctuated with the waves of bombing. If this is so then it is a secondary effect of increased sympathetic tonus and output of adrenaline, a mechanism directly concerned with the instinct of self-preservation. The thyroid gland is nearly always enlarged, and the enlargement is uniform and diffuse. Microscopically it shows a proliferation of the epithelium, often in several layers in the tubules, the colloid is liquefied, there are scattered clusters of lymphatic cells, and the blood supply is increased, but this histological picture is subject to wide variation. The most constant and important symptom of Graves' Disease is the increased rate of general metabolism. Indeed, all the biological reactions are intensified. The body requires more oxygen to perform the same amount of work. This need, which is associated with an excessive excitability of the autonomic nervous system, is met by imposing increased strain on the circulatory system, resulting in tachycardia and palpitation and secondary damage to the myocardium.

Further changes are sometimes shown by disturbances in the metabolism of sugar and fats. Alimentary glycosuria is fairly common. This output of sugar can be increased by means of adrenaline. In a small number of cases there is frank diabetes. Where these factors are present surgical procedures should be undertaken with added caution. Apart from environmental influences enlargement of the thyroid may occur from intrinsic causes due to physiological strain, such as puberty, menstruation, pregnancy, and lactation.

Attempts have been made to classify thyroid disease upon the morphology of the gland, but no satisfactory correlation has been established. The thyroid gland is not necessarily the sole cause of Graves' Disease. Some writers consider that the causes of hyperthyroidism, especially that of Toxic Goitre, lie outside the gland, and are of polyglandular origin. It is not unknown for the removal of a non-toxic adenoma to be followed by the symptoms of marked hyperthyroidism.

The next consistent secondary effect of an endocrine nature in hyperthyroidism is depression of the reproductive function. Menstrual disorders are fairly common. The periods become irregular and may cease, the ovaries finally becoming cystic and degenerate. This is not surprising, for it seems to be a fairly general rule for the gonads to suffer sooner or later in all forms of major endocrine disorder.

\section{Myxoedema.}

Myxoedema may be considered as the counterpart of Graves' Disease. It was first described by
Gull in I873, and its manifestations depend on whether it originates early or late in life. In common with Graves' Disease it is mainly confined to women. Its main symptoms are lethargy and sluggishness of the physical and mental processes. The excitability of the autonomic nervous system is depressed. All these signs are in direct contrast to hyperthyroidism.

The skin is dry and puffy, the hair brittle and tending to fall out, the limbs swell and pads' of fat develop over the supraclavicular fossae. The thyroid gland is absent or atrophic and cannot be felt. Microscopically there is a loss of gland tissue at the expense of connective tissue. Rarely, what there is of the gland is found in an ectopic position along the course of its embryonic duct leading from the foramen caecum at the base of the tongue. The pulse is slow, the blood pressure tends to be subnormal, and the heart may be enlarged. Vaso-motor reaction is depressed, and there is a feeling of coldness in the extremities; the temperature is subnormal, basal metabolism is lower than in any other disease, and the sugar tolerance is raised. In both sexes there is a loss of libido, the sex organs atrophy, leading to amenorrhoea and sterility in women.

Treatment by thyroid extract is specific and constitutes one of the earliest and most satisfactory forms of organotherapy.

\section{Cretinism.}

The thyroid gland may be congenitally absent or may atrophy at an early age, and in some parts of the world cretinism is endemic. In any case all the signs of myxoedema are present, including abnormalities in genital and mental development, but the outstanding feature is lack of growth. These children remain dwarfs, and in appearance show a reversion to the anthropoid type, with bandy legs, the body bent forward, the abdomen pot-bellied, the chin thrust forward with depression at the root of the nose. The centres of ossification of the long bones appear late, and there is delayed union of the epiphyses.

\section{Interglandular Reaction}

Although the thyroid presents such a concise clinical picture, its interglandular reactions are less apparent, for it forms but one link in the chain of interglandular co-ordination, and we cannot separate its effects or be sure that they are direct or mediated through other glands. Like the other glands the thyroid is subservient to pituitary control, and after hypophysectomy in animals it undergoes involution, and the symptoms due to this hypoplasia can be restored by thyroid extract. The pituitary, therefore, exercises a thyreotropic effect which increases thyroid activity. Con- 
yersely thyroidectomy in the rabbit causes hypertrophy of the pituitary. The pituitary-thyroid mechanism controls growth and general metabolism. It is not possible to separate these functions for they fail independently. Clinically, although hyperthyroidism has been described in conjunction with gigantism, the more usual condition of acromegaly shows a shift towards myxoedema. Acromegaly develops slowly, and I have seen a patient of this type from whom a diffuse nodular goitre had been removed. At the age of three she developed infantile paralysis resulting in paralysis of the whole of the left arm, but the interesting feature is that in spite of nervous decontrol the overgrowth of the fingers and hand have not been impaired.

In Cushing's Syndrome due to basophil adenoma of the pituitary there are marked secondary symptoms in metabolism, especially that of fat. In appearance these patients accord more to the hypo than the hyperthyroid type, but in the later stages, or if molested by operation, they are apt to develop a rapid pulse and become anxious. A similar condition obtains after unilateral adrenalectomy in the more advanced cases of virilism. Both these conditions respond well to Lugol's solution. In those rare children who develop isosexual precocity there seems to be a gross upset of this pituitary-thyroid mechanism, which results in lack of growth associated with marked maturity. The paradox is that this condition may be caused either by hypothalamic tumour or adrenal carcinoma. In the latter hyaline changes are found in the cells of the pituitary.

With regard to the gonads, the thyroid may enlarge during the times of stress in the development of the reproductive system. Both the active and deficient functions of the thyroid are associated with depression of sex functions. Experimentally, castration in the dog, rabbit and rat leads to slow involution of the thyroid. Clinically, this was suggestive in a patient who presented himself several years after castration performed abroad.

Patients suffering from hypogonadism have a tendency to be tall, and their epiphyses close late. One of my early intersex patients is growing up with a strong tendency towards acromegaly.

It is doubtful if the thymus gland exerts any endocrine effect, but it is of importance in thyroid surgery. In hyperthyroidism the thymus may be enlarged, and the cause of sudden respiratory failure at operation. After thyroidectomy it undergoes involution. If tadpoles are fed on thymus their metamorphosis is slowed and they. develop into giant tadpoles.

Experimental evidence on the thyro-pancreatic relationship are indefinite.
With regard to thyro-adrenal effects the evidence is conflicting. Experimental results indicate that the adrenal cortex inhibits thyroid activity, and where there is an insufficiency of cortex hyperthyroidism ensues. When thyroid is given in the hypocortical state adrenal failure follows. My own experience confirms that unilateral adrenalectomy in marked virilism induces a hyperthyroid like condition, which is controlled by Lugol's solution.

With regard to the adrenal medulla it is generally agreed that thyroid administration increases the sensitivity to adrenaline, and this effect is most marked on the sympathetic. It is probable that the exopthalmos and other signs of hyperthyroidism are due to this cause.

There are those who consider that hyperthyroidism is due to lack of adrenal cortex, and those who consider overfunctioning of the adrenal medulla plays an important part in the genesis of Graves' Disease. This problem is one of practical importance because it forms the basis of the Goetsch test, for studying the effect of the injection of adrenaline into the hyperthyroid patient.

Adrenaline tumours of the medulla, although they produce an anxious and apprehensive outlook during their hypertensive attacks, do not cause the widespread sympathetic release of hyperthyroidism, but exert their constrictive effects more locally on the blood vessels. These tumours may produces mild degrees of cortical activity, and when this iso present it is of considerable help in the diagnosis.

\section{THE CHRONIC RHEUMATIC DISEASES (Part II)}

By Ernest Fletcher, M.A., M.D., M.R.C.P., and E. Lewis-Faning, B.Sc.Econ., Ph.D.

\section{Clinical Points in Joint Examination}
I. Pain
2. Swelling
3. Tenderness
4. Stiffness
5. Limitation of movement
6. Crepitus
7. Muscle wasting
8. Deformity
9. Oedema

Io. Involvement of surrounding structures.

In this section the two types infective arthritis and osteoarthritis will not be considered separately, but the various points will be brought out in the discussion of the symptom or physical sign. 